\title{
Análisis del perfil psicomotor en infantes colombianos de 4-9 años
}

\author{
Felipe Augusto Reyes-Oyola, Ph. D. ${ }^{a}$ \\ Universidad del Tolima, Colombia \\ Constanza Palomino, Ph. D. ${ }^{b}$ \\ Universidad del Tolima, Colombia \\ Guillermo Meza-Salcedo, Mag. ${ }^{c}$ \\ Corporación Universitaria Minuto de Dios, Colombia
}

fareyeso@ut.edu.co

\section{Resumen (analítico)}

Las alteraciones y trastornos psicomotrices en la infancia pueden repercutir en el desarrollo de habilidades motoras complejas e influir en los aprendizajes escolares. El objetivo del presente estudio fue analizar el perfil psicomotor en población infantil de planteles educativos de Colombia. Se utilizó un diseño transversal, descriptivo; se aplicó la batería psicomotora de Da Fonseca a 131 escolares (56.5\% varones; $43.5 \%$ niñas) de 4-9 años, en educación preescolar y primaria. Los resultados indican que el $76.3 \%$ tiene perfil normal, pero en la praxia fina predominó la dispraxia. Las niñas mostraron mejores valores que los niños en la mayoría de los factores. En conclusión, la mayoría de los infantes presenta un perfil eupráxico, hallándose mejores resultados en las niñas. Esto sugiere la implementación de programas escolares individualizados, previniendo posibles alteraciones.

\section{Palabras clave}

Perfil, infancia, desarrollo, psicomotor.

\section{Thesauro}

DeCS Thesaurus.

\section{Para citar este artículo}

Reyes-Oyola, F. A., Palomino, C, \& Meza-Salcedo, G. (2021). Análisis del perfil psicomotor en infantes colombianos de 4-9 años. Revista Latinoamericana de Ciencias Sociales, Niñez y Juventud, 19(2), 1-17. https://dx.doi.org/10.11600/rlcsnj.19.2.4193

\section{Historial}

Recibido: 29.04 .2020

Aceptado: 02.07.2020

Publicado: 30.04 .2021

\section{Información artículo}

Este artículo de investigación científica y tecnológica hace parte del proyecto denominado Perfil psicomotor en infantes de educación básica primaria pertenecientes a instituciones educativas oficiales de Ibagué (Colombia), perteneciente al grupo de investigación Formación en Movimiento, de la Universidad del Tolima, desarrollado en alianza con el grupo de investigación Infancias y Saberes, de la Corporación Universitaria Minuto de Dios. Estudio cuantitativo, transversal y descriptivo. Se realizó entre el 01 de Febrero y el 30 de Octubre de 2019. Área: educación general. Subárea: educación especial. 


\section{Analysis of psychomotor profile among Colombian children aged 4 to 9 years}

Abstract (analytical)

Psychomotor disturbances and disorders during childhood can have an impact on the development of complex motor skills and influence a child's learning in school. The goal of the present study was to analyze the psychomotor profile of a child population in schools in Colombia. A descriptive and cross-sectional design was used and the Da Fonseca psychomotor battery was applied to 131 students (56.5\% boys; $43.5 \%$ girls) aged $4-9$ years studying in kindergarten and primary education. The results show that $76.3 \%$ have a normal pyschoomotor profile, but Dyspraxia was dominant in Fine Praxia. Girls scored higher than boys in most of the factors. In conclusion, the majority of children present a Eupraxic profile, with improved results among girls. These results can contribute to the implementation of individualized school programs that could prevent possible disturbances.

Keywords

Profile, childhood, development, psychomotor

\section{Análise do perfil psicomotor em crianças colombinas de 4-9 anos}

Resumo (analítico)

A alterações e distúrbios psicomotores na infância podem ter um impacto no desenvolvimento de habilidades motoras complexas e influenciar a aprendizagem escolar. O objetivo do presente estudo foi analisar o perfil psicomotor em uma população infantil em escolas da Colômbia. Utilizou-se delineamento descritivo e transversal. A bateria psicomotora Da Fonseca foi aplicada a 131 estudantes (56.5\% meninos; 43.5 \% meninas) com idades entre 4 e 9 anos, no jardim de infância e no ensino fundamental. Os resultados indicam que $76.3 \%$ têm um perfil normal, mas na praxia fina predominou a dispraxia. As meninas apresentaram melhores valores que os meninos na maioria dos fatores. Em conclusão, a maioria das crianças apresenta um perfil eupráxico, encontrando melhores resultados nas meninas. Isso sugere a implementação de programas escolares individualizados, evitando possíveis distúrbios.

Palavras-chave

Perfil, infância, desenvolvimento, psicomotor.

Información autores

[a] Licenciado en Educación Física, Deportes y Recreación. Especialista en Pedagogía. Magíster en Educación, Universidad del Tolima. Doctor en Investigación de la Enseñanza y el Aprendizaje de Ciencias de la Actividad Física y Deportiva, Universidad de Extremadura. Profesor de la Licenciatura en Pedagogía Infantil, Corporación Universitaria Minuto de Dios, y de la Licenciatura en Educación Física, Deportes y Recreación, Universidad del Tolima. (iD) 0000-0002-8259-0969. H5: 2. Correos electrónicos: freyesoyola@uniminuto.edu.co

fareyeso@ut.edu.co

[b] Licenciada en Educación Física, Deportes y Recreación, Universidad del Tolima. Magíster en Pedagogía de la Cultura Física, Universidad Pedagógica y Tecnológica de Colombia. Doctora en Ciencias de la Actividad Física y el Deporte, Universidad Pablo de Olavide. Profesora de la Licenciatura en Educación Física, Deportes y Recreación, Universidad del Tolima. (iD) 0000-0001-6883-9441. H5: 5. Correo electrónico: cpalominod@ut.edu.co

[c] Licenciado en Teología Pastoral, Universidad Pontificia Bolivariana. Magíster en Filosofía, Universidad Santo Tomás. Profesor de la Licenciatura en Pedagogía Infantil, Corporación Universitaria Minuto de Dios. (iD) 0000-0001-9707-6519. H5: 3. Correo electrónico: guillermo.meza@uniminuto.edu 


\section{Introducción}

$\mathrm{C}_{\text {que surgen cambios progresivos en su habilidad de interactuar con el medio a }}$ través del movimiento (Payne \& Isaacs, 2017). Este es un proceso gradual y continuo en el que se identifican etapas o estadios de creciente nivel de complejidad, que inician con la concepción y culminan en la madurez, con una secuencia similar, pero con un ritmo variable (Vericat \& Orden, 2013), cuyo perfeccionamiento motriz depende de la maduración y del aprendizaje (Gil-Madrona et al., 2008). De este modo, antes de que los niños puedan caminar y luego correr, inicialmente deben aprender a sentarse, gatear y ponerse de pie, primero con apoyo y luego en forma independiente. Así, Patiño (2009) afirma que «el desarrollo psicomotor juega un papel primordial en la vida de un ser humano, desde que el bebé abre sus ojos, su cuerpo se entrega al mundo: de la expresión corporal, de la sensibilidad y, por ende, al de la psicomotricidad» (p. zo).

Es esencial considerar que la evolución de los aspectos psicomotores en niños y niñas varía y no existen unas etapas y tiempos evolutivos estandarizados y rígidos; es decir, cada infante presenta unos ritmos en su crecimiento y su maduración, lo cual depende de la experiencia de cada ser en relación con su cuerpo, el entorno y la relación con las personas (Aucouturier, 2004). Sin embargo, es importante destacar que las diversas investigaciones y estudios realizados a través del tiempo han permitido concretar unos rangos de tiempo cronológico para la adquisición de determinadas habilidades. Un claro ejemplo se presenta en la adquisición de la marcha, que puede variar entre los gy los 16 meses, sin que tenga ninguna relación con la capacidad motriz o intelectual futura (Poó, 2006).

Con base a los anteriores fundamentos, Vericat y Orden (2013) plantean un desarrollo psicomotor normal y patológico. El primero se refiere al proceso que permite al niño adquirir habilidades adecuadas para su edad; mientras que el segundo, siguiendo la perspectiva de Poó (2006), implica apartarse de una manera significativa de lo esperado para la edad. De este modo, el desarrollo psicomotor puede presentar variantes o alteraciones. El 
retraso psicomotor, los diferentes tipos de trastornos del desarrollo y los problemas inaparentes del desarrollo son ejemplos de este tipo de alteraciones, los cuales, desde la perspectiva de la formación docente, requieren de unos saberes disciplinares para su detección y atención (Robledo-Castro et al., 2019).

En contraste, Martín (2013) establece que cuando el proceso de maduración psicomotora se ve alterado, aparecen los trastornos psicomotores, evidenciándose falta de precisión en los movimientos, torpeza o incoordinación en la utilización del cuerpo o en sus relaciones con los objetos. De esta forma, las alteraciones motrices en la población infantil pueden repercutir en el desarrollo de habilidades motoras de mayor complejidad o, en algunos casos, en los procesos de aprendizaje (Pinos-Medrano et al., 2017; Vidarte \& Orozco, 2015). En este sentido, algunos estudios recientes con población infantil han constatado la relación que puede existir entre el desarrollo motriz y el rendimiento académico (Ruiz-Pérez et al., 2016).

De igual manera, los infantes pueden presentar dificultades en el desarrollo de habilidades motoras, incluso aquellos que tienen niveles intelectuales adecuados, sin que estas tengan una explicación médica explícita y concreta desde un trastorno mental, neurológico, congénito o adquirido, un trastorno generalizado del desarrollo o un déficit sensorial visual o auditivo diagnosticable (Salamanca et al., 2016).

De ahí que los primeros años de vida del niño, vinculados principalmente al entorno familiar y escolar, constituyen etapas vitales de desarrollo en el que el niño adquiere el dominio de una serie de habilidades motrices que en un futuro configuran su madurez global (Noguera et al., 2013), partiendo de conseguir el dominio y control del propio cuerpo hasta obtener todas las posibilidades de acción (Gil-Madrona et al., 2008; Santos, 2012). Además, un buen desarrollo psicomotor en la primera infancia es la base de un desarrollo mental posterior, puesto que en los primeros años de vida se promueve el desarrollo intelectual, afectivo y social del niño (Mas et al., 2018).

De este modo, es importante valorar el desarrollo motriz del infante desde su nacimiento (Avilés et al., 2018), así como conocer el perfil y las características psicomotoras en la población infantil; esto con el fin de elaborar estrategias adecuadas de intervención educativa y terapéutica, ajustada a sus necesidades (Delgado \& Montes, 2017), y ayudando a comprender posibles problemas de comportamiento y de aprendizajes (Da Fonseca, 2008).

Los argumentos descritos dan pie para realizar una adecuada evaluación del desarrollo y competencia motora (Stodden et al., 2008), convirtiéndose en una estrategia de salud 
de gran interés en la población infantil, dado que permite detectar tempranamente problemas psicomotrices que pueden dificultar la calidad de vida en edad adulta de la persona (Ayán et al., 2019; Cools et al., 2009), identificándose el grado de eficacia con el que los niños ejecutan las diversas habilidades motrices básicas (Morley et al., 2018).

De acuerdo con lo anterior, surge el objetivo principal del estudio: analizar el perfil psicomotor en infantes colombianos de 4 a 9 años pertenecientes a instituciones educativas de la ciudad de Ibagué. Como objetivos complementarios, se estableció: comparar los resultados obtenidos en función del género y comparar los resultados del presente estudio con otras investigaciones.

\section{Método}

El presente estudio se desarrolló bajo un enfoque metodológico cuantitativo (Abero et al., 2015; Hernández et al., 2014; Niño, 2011), enmarcado en un diseño no experimental, de corte transversal y tipo de estudio descriptivo (Hernández et al., 2014).

\section{Muestra}

Se tomó una muestra por conveniencia, constituida por 131 infantes, con edades comprendidas entre los 4 y 9 años $(5.52 \pm 1,09)$, pertenecientes a los niveles de escolaridad de preescolar, primero, segundo, tercero y cuarto grado de educación básica primaria, de 15 establecimientos educativos del área urbana de la ciudad Ibagué. Las características de la muestra (tabla 1) evidencian que un $56.5 \%$ corresponde a escolares masculinos y el restante $43.5 \%$ a escolares femeninas; además, la mayor cantidad de escolares tienen 6 años (37.4\%) y la mayoría se encuentran en educación preescolar (48.9\%).

Los criterios de inclusión para el estudio fueron: tener la edad destinada para el estudio y portar el uniforme de educación física. Los criterios de exclusión adoptados fueron: no poseer el consentimiento informado y firmado por el padre de familia y tutor, y presentar alguna enfermedad o problema físico. 


\section{Tabla 1}

Características generales de la muestra estudiada

\begin{tabular}{lccc}
\hline \multirow{2}{*}{ Variables } & \multicolumn{2}{c}{ Frecuencia } \\
& & $\mathbf{n}$ & $\%$ \\
\hline \multirow{2}{*}{ Género } & Femenino & 57 & 43.5 \\
& Masculino & 74 & 56.5 \\
\hline & 4 & 25 & 19.1 \\
& 5 & 39 & 29.8 \\
Edad (años) & 6 & 49 & 37.4 \\
& 7 & 12 & 9.2 \\
& 8 & 4 & 3.1 \\
& 9 & 2 & 1.5 \\
Nivel de & Preescolar & 64 & 48.9 \\
escolaridad & Segundo & 12 & 38.2 \\
(grados) & Tercero & 1 & 9.2 \\
& Cuarto & 4 & 3.1 \\
\hline
\end{tabular}

\section{Instrumento}

Como instrumento principal de obtención de datos se utilizó la batería psicomotora (BPM) de Da Fonseca, destinada al estudio del perfil psicomotor del niño. La BPM constituye un instrumento completo para el análisis del desarrollo motor en el niño, dada la diversidad de componentes que toma. Es fiable para valorar e identificar alteraciones psicomotrices en niños y niñas de edades entre los 4 a 12 años, constatado en un estudio que caracterizó el perfil psicomotor en 846 niños colombianos, mostrando un valor aceptable de confiabilidad estadística, luego de obtener un valor de 0.51 al realizar el respectivo análisis de consistencia interna media el Alfa de Cronbach (Vidarte et al., 20og).

La BPM es un instrumento de observación, basado en un «conjunto de tareas que permite detectar déficits funcionales (o su ausencia) en términos psicomotrices, cubriendo la integración sensorial y perceptiva que se relaciona con el potencial de aprendizaje del niño» (Da Fonseca, 2008, p. 108). La batería fue diseñada a partir del modelo de Luriano, en el que relaciona de forma detallada los factores psicomotrices y las unidades funcionales, tanto en los factores como en los subfactores. El nivel de realización es medido numéricamente de la siguiente forma: 1) apraxia; 2) dispraxia; 3) eupraxia; y 4) hiperpraxia (Da Fonseca, 2008, p. 113). En la tabla 2 se observa la relación de unidades funcionales, factores, subfactores y cantidad de tareas o actividades a realizar. 


\section{Tabla 2}

Unidades funcionales, factores, subfactores y tareas de la BPM

\begin{tabular}{|c|c|c|c|}
\hline Unidad & Factores & Subfactores & Tareas \\
\hline \multirow{8}{*}{1} & \multirow{5}{*}{ Tonicidad } & Extensibilidad & $\begin{array}{l}\text { Miembros inferiores: (1) aductores, (2) extensores de } \\
\text { rodilla, (3) cuádriceps femoral. } \\
\text { Miembros superiores: (1) deltoides anterior y pectoral, } \\
\text { (2) flexores de antebrazo, (3) extensores de muñeca. }\end{array}$ \\
\hline & & Pasividad & (1) Miembros inferiores, (2) miembros superiores \\
\hline & & Paratonía & (1) Miembros inferiores, (2) miembros superiores \\
\hline & & Sincinesia & (1) Prueba de comprimir y descomprimir pelota \\
\hline & & Diadococinesias & (1) Prueba de marionetas \\
\hline & \multirow{3}{*}{ Equilibrio } & Inmovilidad & (1) Posición orto-estática \\
\hline & & Equilibrio estático & $\begin{array}{l}\text { (1) Apoyo rectilíneo, (2) equilibrio en la punta de los } \\
\text { pies, (3) apoyo unipedal. }\end{array}$ \\
\hline & & Equilibrio dinámico & $\begin{array}{l}\text { (1) Marcha controlada, (2) evolución en el listón, (3) } \\
\text { salto unipedal, (4) salto a pies juntos }\end{array}$ \\
\hline \multirow{13}{*}{2} & \multirow{4}{*}{ Lateralidad } & Ocular & $\begin{array}{l}\text { (1) Ver a través del tubo, (2) ver a través del orificio en } \\
\text { el centro de la hoja. }\end{array}$ \\
\hline & & Auditiva & (1) Escuchar reloj de cuerda, (2) atender el teléfono \\
\hline & & Manual & (1) Simular escribir, (2) simular cortar \\
\hline & & Pedal & (1) Paso de gigante, (2) simular ponerse los pantalones \\
\hline & \multirow{4}{*}{$\begin{array}{l}\text { Noción del } \\
\text { cuerpo }\end{array}$} & Sentido kinestésico & (1) Toque táctil \\
\hline & & $\begin{array}{l}\text { Reconocimiento derecha } \\
\text { izquierda }\end{array}$ & (1) Prueba output-input \\
\hline & & Auto-imagen & (1) Prueba de autoimagen \\
\hline & & Imitación de Gestos & (1) Prueba de imitación de gestos \\
\hline & \multirow{5}{*}{$\begin{array}{l}\text { Estructuración } \\
\text { espacio- } \\
\text { temporal }\end{array}$} & Dibujo del cuerpo & (1) Dibujar el propio cuerpo \\
\hline & & Organización & (1) Prueba de organización \\
\hline & & Estructuración dinámica & (1) Prueba de estructuración dinámica \\
\hline & & Representación topográfica & (1) Prueba de desplazamiento a partir de un plano \\
\hline & & Estructuración rítmica & (1) Prueba de estructuración rítmica. \\
\hline \multirow{7}{*}{3} & \multirow{4}{*}{ Praxia global } & Coordinación óculo-manual & (1) Lanzamiento de pelota \\
\hline & & Coordinación óculo-pedal & (1) Pateo de pelota \\
\hline & & Dismetría & (1) Observación de las dos tareas anteriores. \\
\hline & & Disociación & $\begin{array}{l}\text { (1) Miembros superiores, (2) miembros inferiores, (3) } \\
\text { coordinación entre miembros superiores e inferiores, } \\
\text { (4) prueba de agilidad }\end{array}$ \\
\hline & \multirow{3}{*}{ Praxia fina } & $\begin{array}{l}\text { Coordinación dinámica } \\
\text { manual }\end{array}$ & (1) Componer y descomponer una pulsera \\
\hline & & Tamborilear & (1)Prueba de tamborilear \\
\hline & & Velocidad-Precisión & (1) Prueba de puntos, (2) prueba de cruces \\
\hline
\end{tabular}

Nota. Elaborada a partir de Da Fonseca (2008). 
Para su análisis, se debe considerar que la puntuación para cada prueba o tarea va de 1 a 4; posterior a esto, se obtiene una puntuación para cada factor. La puntuación máxima de la BPM es de 28 puntos ( 4 x 7 factores) y la mínima de 7 puntos ( 1 x 7 factores), siendo la media 14 puntos. De acuerdo con esto, se construye una escala con los siguientes valores y clasificaciones: 27-28 puntos, superior; 22-26, bueno; 14-21, normal; 9-13, dispráxico; y 7-8, deficitario (Da Fonseca, 2008).

A partir de lo anterior, las categorías de superior y bueno corresponden a un perfil psicomotor hiperpráxico; la categoría de normal, al perfil eupráxico; la categoría de dispráxico a un perfil dispráxico; y la categoría de deficitario, al perfil apráxico.

Las variables analizadas en la investigación fueron las siguientes:

- Sociodemográficas: género (cualitativa, nominal), edad (cuantitativa, escala), nivel de escolaridad (cualitativa, ordinal).

- Psicomotoras: tonicidad, equilibrio, lateralidad, noción del cuerpo (cualitativa, ordinal), estructuración espacio-temporal, praxia global y praxia fina. Todas de tipo cualitativa, ordinal.

\section{Procedimiento y análisis de datos}

El procedimiento para recolectar la información se ilustra en la figura 1. Es importante mencionar que en las instituciones educativas seleccionadas, se realizó la presentación del estudio y se gestionaron los permisos de los rectores, coordinadores y maestros. Seguidamente, se entregó a los infantes el consentimiento informado y fue recolectado después de la autorización y firma de los padres o acudientes.

Paralelamente, se realizó la capacitación del equipo de trabajo y, luego de tener los consentimientos informados, se procedió a realizar una prueba piloto con 15 niños, en aras de identificar y corregir posibles fallas tras ejecutar las actividades y tareas motrices del instrumento.

Posteriormente, se aplicó la BPM a los niños y niñas de los establecimientos educativos seleccionados, atendiendo a un cronograma de trabajo diseñado con anterioridad. Al momento de recolectar los datos, en primer lugar, se tomó la edad, el sexo, el nivel de escolaridad y el nombre del establecimiento educativo. Por su parte, las actividades y tareas motrices de la BPM siguieron el siguiente orden en su realización y observación: tonicidad, equilibrio, lateralidad, noción del cuerpo, estructuración espacio-temporal, praxia global y praxa fina. Los datos fueron consignados en una hoja de registro. 
Figura 1

Procedimiento para recolectar la información

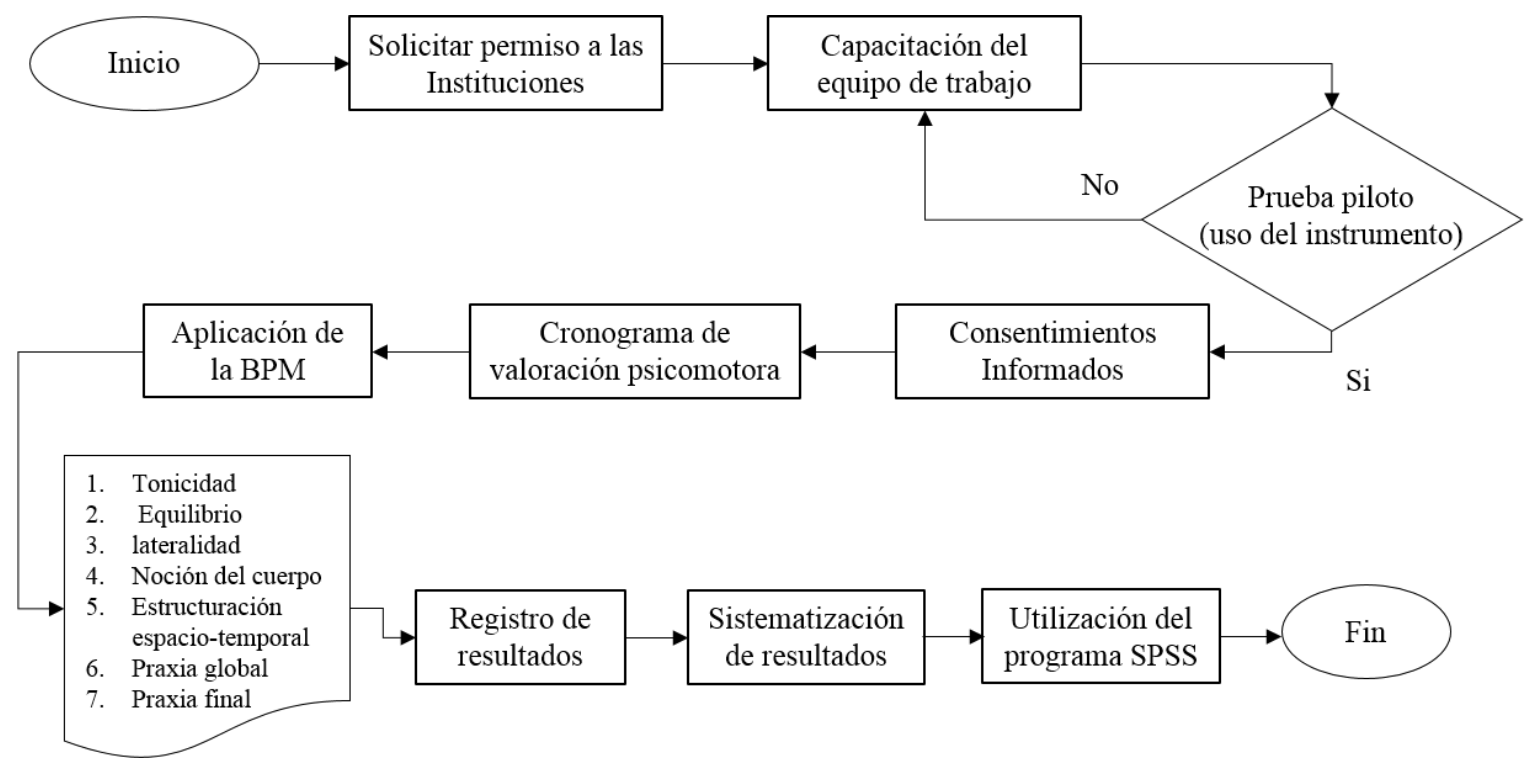

Por su parte, las consideraciones éticas y legales de la investigación se sustentaron bajo la Resolución oo843o del Ministerio de Salud de Colombia (1993) y las normas deontológicas reconocidas por la declaración de Helsinki, que regula las investigaciones realizadas con humanos (Asociación Médica Mundial, 2017).

La información recolectada fue registrada a una hoja de cálculo de Microsoft Excel ${ }^{\mathrm{TM}}$, para luego ser ingresada al programa estadístico IBM SPSS ${ }^{\mathrm{TM}}$ (versión 23). Según los objetivos trazados para el estudio, se realizaron los respectivos análisis descriptivos e inferenciales. En este sentido, los procedimientos descriptivos incluyeron distribución de frecuencias, medidas de tendencia central (media) y medidas de variabilidad (desviación estándar). Respecto a los análisis inferenciales, se empleó la prueba t de student para dos muestras independientes.

\section{Resultados}

Los resultados generales del perfil psicomotor en la muestra estudiada se obtuvieron atendiendo la interpretación de los resultados de la BPM, enunciados por Da Fonseca (2008). En este sentido, el 76.3\% ( $\mathrm{n}=100$ ) de los escolares poseen un perfil normal, el 22.9\% 
$(\mathrm{n}=30)$ tiene un perfil bueno y solo el $0.8 \%(\mathrm{n}=1)$ está en el perfil dispráxico. Se debe resaltar que no hubo niños o niñas en perfiles superiores y deficitarios.

Al realizar un análisis por cada factor psicomotriz (tabla 3) en todos los evaluados se evidencia una mayor prevalencia del perfil psicomotor eupráxico en los factores de tonicidad (75.6\%), equilibrio (63.4\%), lateralidad (50.4\%), noción del cuerpo (65.6\%), estructuración espacio temporal (60.3\%) y praxia global (52.7\%). Por su parte, en el factor de praxia fina predominó el perfil dispráxico (45.8\%) (tabla 3). Estos resultados también se ven reflejados al analizar los perfiles por género, excepto en la lateralidad de las escolares femeninas, donde predominó el perfil hiperpráxico.

Tabla 3

Frecuencia y porcentaje de los perfiles psicomotores; análisis por factor

\begin{tabular}{|c|c|c|c|c|c|c|c|c|c|}
\hline \multirow{3}{*}{$\begin{array}{l}\text { Factores } \\
\text { psicomotrices }\end{array}$} & \multirow{3}{*}{ Género } & \multicolumn{8}{|c|}{ Perfiles psicomotores } \\
\hline & & \multicolumn{2}{|c|}{ Apráxico } & \multicolumn{2}{|c|}{ Dispráxico } & \multicolumn{2}{|c|}{ Eupráxico } & \multicolumn{2}{|c|}{ Hiperpráxico } \\
\hline & & $\mathbf{n}$ & $\%$ & $\mathbf{n}$ & $\%$ & $\mathbf{n}$ & $\%$ & $\mathbf{n}$ & $\%$ \\
\hline \multirow{3}{*}{ Tonicidad } & $T \lambda$ & 0 & 0 & 25 & 19.1 & 99 & 75.6 & 7 & 5.3 \\
\hline & FЖ & 0 & 0 & 8 & 14 & 47 & 82.5 & 2 & 3.5 \\
\hline & $\mathrm{M} \triangle$ & 0 & 0 & 17 & 23 & 52 & 70.3 & 5 & 6.8 \\
\hline \multirow{3}{*}{ Equilibrio } & $T \lambda$ & 0 & 0 & 24 & 18.3 & 83 & 63.4 & 24 & 18.3 \\
\hline & FЖ & 0 & 0 & 8 & 14 & 36 & 63.2 & 13 & 22.8 \\
\hline & $M \triangle$ & 0 & 0 & 16 & 21.6 & 47 & 63.5 & 11 & 14.9 \\
\hline \multirow{3}{*}{ Lateralidad } & $T \lambda$ & 1 & 0.8 & 5 & 3.8 & 66 & 50.4 & 59 & 45 \\
\hline & FЖ & 0 & 0 & 4 & 7 & 24 & 42.1 & 29 & 50.9 \\
\hline & $\mathrm{M} \triangle$ & 1 & 1.4 & 1 & 1.4 & 42 & 56.8 & 30 & 40.5 \\
\hline \multirow{3}{*}{ Noción del cuerpo } & $T \lambda$ & 0 & 0 & 19 & 14.5 & 86 & 65.6 & 26 & 19.8 \\
\hline & FЖ & 0 & 0 & 9 & 15.8 & 33 & 57.9 & 15 & 26.3 \\
\hline & $M \triangle$ & 0 & 0 & 10 & 13.5 & 53 & 71.6 & 11 & 14.9 \\
\hline \multirow{3}{*}{$\begin{array}{l}\text { Estructuración } \\
\text { espacio-temporal }\end{array}$} & $T \lambda$ & 0 & 0 & 50 & 38.2 & 79 & 60.3 & 2 & 1.5 \\
\hline & FЖ & 0 & 0 & 21 & 36.8 & 34 & 59.6 & 2 & 3.5 \\
\hline & $\mathrm{M} \triangle$ & 0 & 0 & 29 & 39.2 & 45 & 60.8 & 0 & 0 \\
\hline \multirow{3}{*}{ Praxia global } & $T \lambda$ & 8 & 6.1 & 44 & 33.6 & 69 & 52.7 & 10 & 7.6 \\
\hline & FЖ & 4 & 7 & 23 & 40.4 & 25 & 43.9 & 5 & 8.8 \\
\hline & $\mathrm{M} \Delta$ & 4 & 5.4 & 21 & 28.4 & 44 & 59.5 & 5 & 6.8 \\
\hline \multirow{3}{*}{ Praxia Fina } & $T \lambda$ & 4 & 3.1 & 60 & 45.8 & 58 & 44.3 & 9 & 6.9 \\
\hline & FЖ & 2 & 3.5 & 25 & 43.9 & 24 & 42.1 & 6 & 10.5 \\
\hline & $\mathrm{M} \triangle$ & 2 & 2.7 & 35 & 47.3 & 34 & 45.9 & 3 & 4.1 \\
\hline
\end{tabular}

Notas. $\lambda$ Todos los escolares. Ж Escolares femeninas. $\Delta$ Escolares masculinos. 
En la tabla 4 se presentan las comparaciones de los factores psicomotores entre grupos diferenciados por género. Así, las escolares femeninas mostraron mejores resultados en todos los factores y en el perfil psicomotor total en comparación con los escolares masculinos, excepto en la praxia global, aunque las diferencias no fueron estadísticamente significativas para ninguna de las circunstancias ( $\mathrm{p}>0.05)$.

\section{Tabla 4}

Factores psicomotores; comparación entre grupos diferenciados por sexo

\begin{tabular}{lcccc}
\hline Variables (factores) & $\begin{array}{c}\text { Todos }(n=131) \\
\mathbf{X} \pm \mathrm{DE} €\end{array}$ & $\begin{array}{c}\text { Niñas }(\mathrm{n}=57) \\
\mathbf{X} \pm \mathrm{DE} €\end{array}$ & $\begin{array}{c}\text { Niños }(\mathbf{n}=\mathbf{7 4}) \\
\mathbf{X} \pm \mathrm{DE} €\end{array}$ & p乏̌ \\
\hline Tonicidad & $2.93 \pm 0.42$ & $2.95 \pm 0.38$ & $2.92 \pm 0.45$ & 0.671 \\
Equilibrio & $3.02 \pm 0.48$ & $3.09 \pm 0.46$ & $2.96 \pm 0.50$ & 0.135 \\
Lateralidad & $3.40 \pm 0.60$ & $3.44 \pm 0.62$ & $3.36 \pm 0.58$ & 0.490 \\
Noción del cuerpo & $3.03 \pm 0.53$ & $3.04 \pm 0.56$ & $3.02 \pm 0.50$ & 0.792 \\
Estructuración espacio & $2.69 \pm 0.47$ & $2.76 \pm 0.53$ & $2.63 \pm 0.41$ & 0.124 \\
temporal & $2.63 \pm 0.65$ & $2.54 \pm 0.68$ & $2.71 \pm 0.62$ & 0.150 \\
Praxia global & $2.59 \pm 0.60$ & $2.61 \pm 0.67$ & $2.57 \pm 0.54$ & 0.768 \\
Praxia fina & $20.31 \pm 2.24$ & $20.45 \pm 2.56$ & $20.19 \pm 1.96$ & 0.516 \\
Perfil psicomotor & & & & \\
\hline
\end{tabular}

Nota. F€: media; „: desviación estándar: ב̌ nivel de significación.

\section{Discusión}

La investigación realizada permitió analizar el perfil psicomotor de los escolares objeto de estudio en los planteles educativos seleccionados de la ciudad de Ibagué (Colombia). En este orden de ideas, el estudio mostró que la mayoría de los niños y niñas examinadas (76.3\%) están en un perfil psicomotor normal o eupráxico, lo que demuestra que su nivel de realización de las tareas fue completo, adecuado y controlado en la mayoría de los factores. De igual forma, no presentaron problemas psicomotrices al no detectarse señales desviadas en la observación, ni tampoco poseen dificultades de aprendizaje significativas o señales de disfunción cerebral mínima (Da Fonseca, 2008).

No obstante, bajo el perfil psicomotor eupráxico, pueden existir factores que revelan inmadurez o imprecisión de control. Es así que una cifra considerable de escolares se encuentra en un perfil dispráxico en los factores de praxia fina (45.8\%), praxia global (33.6\%) y 
estructuración espacio-temporal ( $38.2 \%)$, demostrando que estos infantes realizaron las actividades con dificultades de control, con combinaciones de señales desviadas y, ante esto, probablemente pueden tener dificultades ligeras de aprendizaje (Da Fonseca, 2008). Estos indicadores incentivan a planificar y realizar actividades que mejoren los componentes motrices de la estructuración espacial y temporal, así como la motricidad fina y gruesa en el infante.

Por su parte, al comparar los resultados del presente estudio con otras investigaciones, se observa que el perfil eupráxico también fue predominante en 800 escolares de los 8 a 12 años de la ciudad de Popayán (Colombia) (Roldán \& Paz, 2013) y 6o escolares de 6 a 8 años de básica primaria, de etnias indígenas Inga y Kamëntsá de Colón, de Putumayo (Colombia) (Lourido et al., 2016). Igualmente, 755 escolares de 4 a 8 años de colegios oficiales y privados reflejaron perfiles psicomotores normales (oficial: $39.7 \%$; privado: $31 \%$ ) y buenos (oficial: 57.5\%; privado: 67.6\%) (Palacio-Duran et al., 2017).

Valores similares se presentaron en un estudio con niños de la ciudad de Barranquilla, donde predominó el perfil eupráxico para el equilibrio (42.9\%), lateralidad (46.3\%), noción del cuerpo (62.5\%), tonicidad (78.1\%), praxia global (49.6\%) y estructuración espacio temporal (50.9\%) (Noguera et al., 2013).

De modo similar, 217 niñas y niños españoles revelaron una media de desarrollo psicomotor del $81.6 \%$ del desarrollo esperado para la edad cronológica y solo una prevalencia del retraso motor del $4 \%$ (Delgado \& Montes, 2017). Se debe aclarar que en el estudio citado se valoró el perfil del desarrollo psicomotor mediante otro instrumento de medición; en este caso, la escala observacional del desarrollo, versión breve.

Por otro lado, cifras contrarias a las anteriores son descritas por Noguera et al. (2013) y Noguera y García (2013) en su estudio realizado con 389 estudiantes de 4 a 8 años de la ciudad de Barranquilla, donde predominó el perfil dispráxico con un $44 \%$, seguido del normal (36\%) y el perfil bueno (19.8\%). Además, un grupo de niños de Popayán mostró un perfil dispráxico en la estructuración espacio-temporal (78.18\%) (Roldán \& Paz, 2013).

En otra línea, al revisar los resultados de todos los escolares ibaguereños de la tabla 4 , se evidencia cifras inferiores en comparación con un estudio en 60 niños y niñas de 6 a 8 años de la ciudad de Barranquilla (Noguera et al., 2015), específicamente en las puntuaciones medias de la tonicidad (2.93 vs. 3.01), el equilibrio (3.02 vs. 3.16), lateralidad (3.40 vs. 3.61), estructuración espacio-temporal (2.76 vs. 2.69), praxia global (2.71 vs. 2.63), praxia fina (3.03 vs. 2.59) y el perfil psicomotor total (21.18 vs. 20.31). Sin embargo, el estudio en 
Ibagué mostró mejores valores en el factor de noción del cuerpo (3.03 vs. 2.86). Se aclara que los infantes de la ciudad de Barranquilla tenían mayor edad que los de Ibagué y una menor muestra.

En cuanto a las comparaciones realizadas por sexo (tabla 4), un estudio realizado en niños españoles de 3 a 6 años revela resultados similares al no identificarse diferencias significativas en las comparaciones por sexo (Martínez-Moreno et al., 2020). Se aclara que en este estudio se determinó el perfil psicomotor mediante el test de habilidades psicomotoras para niños (séptima edición).

Las comparaciones realizadas con otros estudios constituyen un importante ejercicio y referente, a fin de identificar resultados similares, mejores o inferiores, de acuerdo con las variables estudiadas. Del mismo modo, el actual estudio sirve como punto de partida para futuras investigaciones, de manera que investigadores opten por profundizar o trabajar alguna variable.

A nivel general, el estudio se realizó satisfactoriamente, contándose con buena actitud y disposición de parte de los escolares seleccionados, apoyado del aval de los rectores, directores y profesores.

En cuanto a nuestras conclusiones, en los escolares estudiados en la ciudad de Ibagué se identificó, en su mayoría, un perfil psicomotor eupráxico; pero, del mismo modo, valores importantes en cuanto a perfiles psicomotores dispráxicos y apráxicos. En este sentido, se deben implementar programas escolares basados en el juego que busquen prevenir y disminuir dificultades y trastornos motores, así como también fortalecer la motricidad, fortaleciendo las capacidades cognitivas, sociales y lingüísticas de los niños.

Estos programas deben ser orientados por docentes o profesionales en el área de la educación física y la pedagogía infantil. En estos, no solo se deben trasmitir conocimientos académicos, sino que se debe guiar a los alumnos y alumnas para que vivan las experiencias de aprendizaje para la vida de manera enriquecedora e innovadora (FernándezMartínez \& Montero-García, 2016).

Es importante tomar como ejemplo el programa de actividades implementado en niñas y niños colombianos, el cual se basó en tareas de habilidades motoras (lanzamiento y recepción de objetos, carreras, saltos y giros), realizadas de modo individual, en parejas y en grupos, durante 24 sesiones, con duración de una hora cada una. Dicho programa, aplicado en el intermedio de una pre-evaluación y posevaluación, mostró mejorías en los puntajes de 6 factores evaluados (Noguera et al., 2015). 
Las principales limitantes del estudio, en primer lugar, se orientan a la extensión del instrumento y el bajo grupo de personal de apoyo para la recogida de información, lo cual pudo influir en el tiempo y la obtención de datos más abundante; y, en segundo lugar, se presentaron diferentes actividades institucionales que retrasaron el trabajo de campo; por consiguiente, hubo varias modificaciones en el cronograma propuesto para la investigación.

Dado lo anterior, como perspectiva para futuros estudios, se propone contar con un equipo de apoyo amplio, para así valorar una muestra mayor de escolares, obteniendo información más amplia y confiable. Ligado a lo anterior, se recomienda analizar otras variables que pueden resultar relevantes, permitiendo un análisis más completo del desarrollo motor del infante.

\section{Agradecimientos}

A los rectores, coordinadores y profesores de las respectivas instituciones educativas por otorgar la autorización y espacio en las sesiones de clase. A los padres de familia o tutores por permitir participar a sus hijos de este estudio. A todos los niños y niñas que participaron en el estudio, gracias a su colaboración, disposición, desempeño y esfuerzo en cada una de las actividades. Finalmente, agradecer al grupo de estudiantes por su valioso tiempo y apoyo en las jornadas de capacitación y el trabajo de campo.

\section{Referencias}

Abero, L., Berardi, L., Capocasale, A., García, S., \& Rojas, R. (2015). Investigación educativa: abriendo puertas al conocimiento. Camus Ediciones.

Asociación Médica Mundial. (2017). Declaración de Helsinki de la AMM: principios éticos para las investigaciones médicas en seres humanos. https://bit.ly/35 $\mathrm{fB} 6 \mathrm{nO}$

Aucouturier, B. (2004). Los fantasmas de la acción. Graó.

Avilés, J., Borrego, F., López, G., \& Díaz, A. (2018). Design and validation of a psychomotor profile evaluation scale in early childhood education. Journal of Human Sport and Exercise, 13, 421-431.

Ayán, C., Cancela, J., Sánchez-Lastra, M., Carballo-Roales, A., Domínguez-Meis, F., \& Redondo-Gutiérrez, L. (2019). Fiabilidad y validez de la batería TGMD-2 en población española. Revista Iberoamericana de Diagnóstico y Evaluación, 1(50), 21-33. https://doi.org/gbcx 
Cools, W., De Martelaer, K., Samaey, C., \& Andries, C. (2009). Movement skill assessment of typically developing preschool children: A review of seven movement skill assessment tools. Journal of Sports Science and Medicine, 8(2), 154-168.

Da Fonseca, V. (2008). Manual de observación psicomotriz. Inde Publicaciones.

Delgado, L., \& Montes, R. (2017). Perfil y desarrollo psicomotor de los niños españoles entre 3 a 6 años. Sportis Scientific Technical Journal of School Sport, Physical Education and Psychomotricity, 3(3), 454-470. https://doi.org/10.17979/sportis.2017.3.3.2002

Fernández-Martínez, A. M., \& Montero-García, I. (2016). Aportes para la educación de la Inteligencia Emocional desde la Educación Infantil. Revista Latinoamericana de Ciencias Sociales, Niñez y Juventud, 14(1), 53-66.

Gil-Madrona, P., Contreras, O., \& Gómez, I. (2008). Habilidades motrices en la infancia y su desarrollo desde una educación física animada. Revista Iberoamericana de Educación, 1(47), 71-96.

Hernández, R., Fernández, C., \& Baptista, P. (2014). Metodología de la investigación. McGraw-Hill.

Lourido, D., Velasco, D., Chalapud, I., Chalpartar, K., \& Rangel, A. (2016). Estudio comparativo del perfil psicomotor en escolares de etnia indígena y colonos de Colón, Putumayo. Fedumar, Pedagogía y Educación, 3(1), 165-181.

Martín, D. (2013). Psicomotricidad e intervención educativa. Pirámide.

Martínez-Moreno, A., Giménez, S., \& Díaz, A. (2020). The psychomotor profile of pupils in early childhood education. Sustainability, 12(6), 1-11. https://doi.org/gbcz

Mas, M., Jiménez, L., \& Riera, C. (2018). Systematization of the psychomotor activity and cognitive development. Psicología Educativa, 24(1), 38-41. https://doi.org/gbc2

Ministerio de Salud de Colombia. (1993). Resolución número 8430 de 1993 (octubre 4). Por la cual se establecen las normas cientificas, técnicas y administrativas para la investigación en salud. https://bit.ly/2PoDgSu

Morley, D., Van Rossum, T., Richardson, D., \& Foweather, L. (2018). Expert recommendations for the design of a children's movement competence assessment tool for use by primary school teachers. European Physical Education Review, 25(2), 524-543. https://doi.org/10.1177/1356336X17751358

Niño, V. (2011). Metodología de la investigación: diseño y ejecución. Ediciones de la U.

Noguera, L., Herazo, Y., \& Vidarte, J. (2013). Correlación entre perfil psicomotor y rendimiento lógico-matemático en niños de 4 a 8 años. Revista Ciencias de la Salud, $11(2), 185-194$. 
Noguera, L., Quintero, M., Vidarte, J., \& García, R. (2015). Efectos de un programa de ejercicios sobre el perfil psicomotor en escolares. Revista Colombiana de Rehabilitación, 14(1), 38-44. https://doi.org/10.30788/revcolreh.v14.n1.2015.17

Noguera, L., \& García, F. (2013). Perfil psicomotor en niños escolares: diferencias de género. Ciencia e Innovación en Salud, 1(2), 108-113. https://doi.org/gbe3

Palacio-Durán, E., Pinillos-Patiño, Y., Herezo-Beltrán, L., Galeano-Muñoz, L., \& PrietoSuárez, E. (2017). Determinantes del desempeño psicomotor en escolares de Barranquilla, Colombia. Revista de Salud Pública, 19(3), 297-303. https://doi.org/gbc4

Patiño, V. (2009). Valoración del estado psicomotor de los niños preescolares del hogar infantil Ormaza de la comuna nor-oriental de Pereira 20o8, mediante el test de Tepsi [Tesis de pregrado]. Universidad Tecnológica de Pereira.

Payne, V., \& Isaacs, L. (2017). Human motor development: A lifespan approach. Routledge.

Pinos-Medrano, V., Medrano-Núñez, N., \& Alarcón-Salvatierra, P. (2017). La dispraxia y sus efectos en el aprendizaje. Revista Dominio de las Ciencias, $3(2), 380-400$.

Poó, P. (2006). Desarrollo psicomotor: características evolutivas de 0-3 años, signos de alerta. V Reunión Anual de la Sociedad Asturiana de Pediatría de Atención Primaria, Barcelona, España.

Roldán, E., \& Paz, A. (2013). Relación de sobrepeso y obesidad con nivel de actividad física, condición física, perfil psicomotor y rendimiento escolar en población infantil (8 a 12 años) de Popayán. Movimiento Científico, 7(1), 71-84. https://doi.org/gbc5

Robledo-Castro, C., Amador-Pineda, L. H., \& Ñáñez-Rodríguez, J. J. (2019). Políticas públicas y políticas educativas para la primera infancia: desafíos de la formación del educador infantil. Revista Latinoamericana de Ciencias Sociales, Niñez y Juventud, ${ }_{17}(1)$, 169-191. https://doi.org/10.1160o/1692715x.17110

Ruiz-Pérez, L. M. R., Navia, J. A., Amengual, A. R., Otero, I. R., \& Palomo, M. (2016). Coordinación motriz y rendimiento académico en adolescentes. Retos: Nuevas Tendencias en Educación Física, Deporte y Recreación, 29, 86-89. https://doi.org/gbc6 Salamanca, L., Naranjo, M., Díaz, L., \& Salinas, R. (2016). Estudio de asociación del trastorno del desarrollo de la coordinación con los problemas de conducta en niños de la ciudad de Bucaramanga, Colombia. Revista de Salud Pública, 19(3), 351-363.

Santos, A. (2012). Consideraciones sobre la psicomotricidad en educación infantil. Revista Vozes dos Vales, 1(1), 1-18.

Stodden, D. F., Goodway, J. D., Langendorfer, S. J., Roberton, M. A., Rudisill, M. E., García, C., \& García, L. E. (2008). A developmental perspective on the role of motor 
skill competence in physical activity: An emergent relationship. Quest, 60(2), 290-306. https://doi.org/10.1080/00336297.2008.10483582

Vericat, A., \& Orden, A. (2013). El desarrollo psicomotor y sus alteraciones: entre lo normal y lo patológico. Ciência \& Saúde Coletiva, 18(10), 2977-2984. https://doi.org/gbc7

Vidarte, J. A., Ezquerro, M., \& Giráldez, M. A. (2009). Perfil psicomotor de niños de 5 a 12 años diagnosticados clínicamente de trastorno por déficit de atención/hiperactividad en Colombia. Revista de Neurología, 49(2), 69-75. https://doi.org/gbc8

Vidarte, J. A., \& Orozco, C. I. (2015). Relaciones entre el desarrollo psicomotor y el rendimiento académico en niños de 5 y 6 años de una institución educativa de La Virginia (Risaralda, Colombia). Revista Latinoamericana de Estudios Educativos, 11(2), 190-204. 